\title{
Unilateral neonatal cerebral infarction in full term infants
}

\author{
Javier Estan, Peter Hope
}

\begin{abstract}
Aims-To determine the prevalence of unilateral neonatal cerebral infarction in infants born at 32 weeks gestation and above; to describe the clinical course, imaging results, and outcome of neonatal cerebral infarction; and to investigate possible aetiology.

Methods-Twelve cases of unilateral neonatal cerebral infarction were identified from neonatal unit records for the years 1987-93. Each case was matched with two controls.
\end{abstract}

Results-All cases of neonatal cerebral infarction occurred in full term infants. The prevalence was around 1 in 4000 , and neonatal cerebral infarction was found in $12 \%$ of infants presenting with neonatal seizures. Cerebral ultrasound scans failed to demonstrate lesions seen by computed tomography in nine of 12 cases. Cases were more likely than controls to require assisted ventilation for resuscitation at birth (OR 7.0, 95\% confidence interval 1.04-53.5), but Apgar scores at 5 minutes were no different. One infant with neonatal cerebral infarction developed a hemiparesis, the other 11 had normal motor development when assessed at 11-60 (median 33) months. None had overt cognitive deficits or persisting seizure disorder. Conclusions-Neonatal cerebral infarction is a relatively common cause of neonatal seizures, but the aetiology remains unclear. Parents need to be made aware of possible neurological sequelae, but most cases in this series had a normal outcome. (Arch Dis Child 1997;76:F88-F93)

Keywords: cerebral infarction; seizures; neurodevelopmental outcome; stroke; hemiplegia.

Unilateral neonatal cerebral infarction, or neonatal stroke, is well recognised and usually presents with neonatal seizures; it is one cause of spastic hemiplegic cerebral palsy. ${ }^{1-3}$ Several studies have shown that it is the cause of a significant minority of neonatal seizures, ${ }^{4-6}$ but this information has not filtered into some standard texts and neonatal handbooks. Neonatal cerebral infarction is sometimes not even listed in tables of the causes of neonatal seizures in these texts, while rarities such as pyridoxine dependency are always mentioned. In clinical practice neonatal cerebral infarction is a relatively common cause of neonatal seizures, often presenting as focal convulsions in an otherwise healthy full term neonate on the postnatal wards. Levy et $a l^{6}$ have suggested
Table 1 Aetiology of seizures in neonates $>31$ weeks gestation

Birth asphyxia
Cerebral infarction
Haemorrhage
Meningitis
Metabolic disorders:
Hypoglycaemia
Pyruvate dehydrogenase deficiency
Non-ketotic hyperglycinaemia
Others:
Kernicterus
Dysmorphic syndromes
Non-immune hydrops
Head trauma
Unknown

Hypoglycaemia

Kernicterus

Non-immune hydrops

Unknown

that neonatal cerebral infarction is the second most common identifiable cause of seizures in full term infants.

Nevertheless, aetiology remains obscure in most cases, and the published data about prognosis are contradictory. ${ }^{37}$ It has been our practice to counsel parents about the likelihood of hemiplegic cerebral palsy when the diagnosis of neonatal cerebral infarction is established in the neonatal period. However, we have recently seen a series of babies with normal neurological outcome after neonatal cerebral infarction has been diagnosed by cerebral ultrasound scans, computed tomography, or both.

This retrospective review was carried out to determine how frequently neonatal seizures in term infants could be attributed to neonatal cerebral infarction in our inborn babies, and to look for any perinatal risk factors that may give a clue to aetiology, in a case-control study. We also looked at the medium term neurodevelopmental outcome following neonatal cerebral infarct.

\section{Methods}

Neonatal unit records for the period 1987-93 were searched to identify all infants of greater than 31 weeks gestation who had presented with neonatal seizures or who had overt seizures while in the unit. Individual patient records were then searched to establish, wherever possible, the aetiology of the seizures, and to identify the subgroup of neonates in whom imaging had revealed neonatal cerebral infarction to be the probable cause. When these cases were identified, controls for each case were selected from the unit register. Two controls of the same sex and gestational age (within one week) were selected for each case by searching the register forward and backward from the date of admission of the index case. The first infant born after the index case and the last infant born before, who fulfilled sex and gestational age criteria and did not have seizures, hypoxic-ischaemic encephalopathy, or confirmed infection, served as controls. 
Table 2 Main clinical features of 12 infants with neonatal cerebral infarction

\begin{tabular}{|c|c|c|c|c|c|c|c|c|c|c|c|c|}
\hline $\begin{array}{l}\text { Case } \\
\text { No }\end{array}$ & $\begin{array}{l}\text { Birthweight } \\
(g)\end{array}$ & $\begin{array}{l}\text { Gestational } \\
\text { age (weeks) }\end{array}$ & Pregnancy & $C T G$ & Amniotic fluid & Delivery & $\begin{array}{l}\text { Arterial } \\
\text { cord } p H\end{array}$ & $\begin{array}{l}\text { Apgar score } \\
(1 \mathrm{~min})\end{array}$ & $\begin{array}{l}\text { Apgar score } \\
(5 \mathrm{~min})\end{array}$ & Resuscitation & $\begin{array}{l}\text { Onset of } \\
\text { fits }(h)\end{array}$ & Type of fits \\
\hline 1 & 3774 & 41 & Normal & $\mathrm{N}$ & Clear & SVD & & 4 & 10 & Face mask & 16 & Right focal \\
\hline 2 & 3564 & 40 & Normal & $\mathrm{N}$ & $\begin{array}{l}\text { Fluid } \\
\text { meconium }\end{array}$ & SVD & 7.18 & 2 & 9 & Intubation & 24 & Subtle \\
\hline 3 & 3021 & 42 & Normal & B & $\begin{array}{l}\text { Thick } \\
\text { meconium }\end{array}$ & LSCS & 7.23 & 8 & 10 & None & 48 & Generalised \\
\hline 4 & 4460 & 41 & Normal & $\mathrm{N}$ & $\begin{array}{l}\text { Thick } \\
\text { meconium }\end{array}$ & SVD & 7.19 & 5 & 9 & Intubation & 76 & Generalised \\
\hline 5 & 3950 & 40 & Normal & DIPS & Clear & LSCS & & 5 & 8 & Intubation & 24 & Left focal \\
\hline 6 & 2541 & 39 & $\mathrm{APH}$ & B & Clear & SVD & 7.24 & 9 & 10 & None & 12 & Generalised \\
\hline 7 & 3468 & 41 & Normal & & $\begin{array}{l}\text { Fluid } \\
\text { meconium }\end{array}$ & LSCS & 7.32 & 2 & 8 & Face mask & 45 & Right focal \\
\hline 8 & 2931 & 37 & Normal & $\mathrm{T}$ & Clear & Forceps & 7.11 & 8 & 10 & None & 28 & Right focal \\
\hline 9 & 3500 & 40 & Normal & & Unknown & Ventouse & & 7 & 9 & None & 36 & Left focal \\
\hline 10 & 3479 & 41 & PET & & Clear & SVD & 7.12 & 9 & 10 & None & 64 & Right focal \\
\hline 11 & 3410 & 41 & Normal & $\mathrm{T}$ & $\begin{array}{l}\text { Thick } \\
\text { meconium }\end{array}$ & Forceps & 7.02 & 4 & 9 & Face mask & 12 & Right focal \\
\hline 12 & 3851 & 41 & Normal & $\mathrm{N}$ & $\begin{array}{l}\text { Fluid } \\
\text { meconium }\end{array}$ & SVD & 7.34 & 9 & 10 & None & 56 & Left focal \\
\hline
\end{tabular}

APH: Antepartum haemorrhage, PET: Pre-eclampsia, B: Bradycardia, DIPS: Type II decelerations, T: Tachycardia, SVD: Spontaneous vaginal delivery, LSCS: Lower segment caesarean section.

Table 3 Imaging results and follow up data of 12 infants with neonatal cerebral infarction

\begin{tabular}{|c|c|c|c|c|c|c|c|c|c|}
\hline $\begin{array}{l}\text { Case } \\
\text { No }\end{array}$ & $\begin{array}{l}\text { Results and timing of } \\
\text { initial ultrasound } \\
\text { scan }\end{array}$ & $\begin{array}{l}\text { Results and timing of } \\
\text { follow up scans }\end{array}$ & $\begin{array}{l}\text { Results and timing of computed } \\
\text { tomography }\end{array}$ & $\begin{array}{l}\text { Result and timing of } \\
\text { EEG }\end{array}$ & $\begin{array}{l}\text { Follow } \\
\text { up } \\
\text { period } \\
\text { (months) }\end{array}$ & $\begin{array}{l}\text { Persistent } \\
\text { seizure } \\
\text { disorder }\end{array}$ & Vision & Hearing & Motor deficit \\
\hline 1 & Normal (d1) & $\begin{array}{l}\text { Normal }(\mathrm{d} 2,3,4,6 \text {, } \\
\text { w9) }\end{array}$ & Left parietal infarction (d3) & $\begin{array}{l}\text { Left asymmetrical } \\
\text { seizure activity (d2) }\end{array}$ & 20 & No & Normal & Normal & No \\
\hline 2 & Normal (d2) & Normal $(\mathrm{d} 3,7)$ & Right temporal infarction (d4) & $\begin{array}{l}\text { Generalised seizure } \\
\text { activity (d3) }\end{array}$ & 60 & No & Normal & Normal & No \\
\hline 3 & Normal (d1) & $\begin{array}{l}\text { Right parietal } \\
\text { echodensity (d3, } 4 \text {, } \\
5, \text { w7) }\end{array}$ & $\begin{array}{l}\text { Right frontoparietal infarction } \\
\text { (d7) }\end{array}$ & Normal (d4) & 34 & No & Normal & Normal & No \\
\hline 4 & Normal (d4) & Normal (d5, 10, w11) & Right parietal infarction (d8) & Normal (d4) & 48 & No & Normal & Normal & No \\
\hline 5 & Normal (d1) & $\begin{array}{l}\text { Right parietal } \\
\text { echodensity (d2, } 8 \text {, } \\
\text { w6) }\end{array}$ & Right parietal infarction (d7) & $\begin{array}{l}\text { Right asymmetrical } \\
\text { seizure activity (d4) }\end{array}$ & 48 & No & Normal & Normal & No \\
\hline 6 & Normal (d2) & Normal (d5, w4) & $\begin{array}{l}\text { Right middle cerebral artery } \\
\text { infarction }(\mathrm{d} 2)\end{array}$ & Normal (d2) & 54 & No & Normal & Normal & $\begin{array}{l}\text { Left } \\
\text { hemiparesis }\end{array}$ \\
\hline 7 & Normal (d2) & Normal (d4, w6) & $\begin{array}{l}\text { Left temporoparietal } \\
\text { infarction }(\mathrm{d} 7)\end{array}$ & Not done & 25 & No & Normal & Normal & No \\
\hline 8 & Normal (d2) & Normal (d3) & Left frontal infarction (d3) & $\begin{array}{l}\text { Left asymmetrical } \\
\text { seizure activity (d3) }\end{array}$ & $14^{\star}$ & No & Normal & Normal & No \\
\hline 9 & Normal (d2) & Normal (d5, 7, w6) & $\begin{array}{l}\text { Infarction-Left middle } \\
\quad \text { cerebral artery territory (d7) }\end{array}$ & Normal (d7) & 11 & No & Normal & Normal & No \\
\hline 10 & $\begin{array}{l}\text { Left parietal } \\
\text { echodensity (d2, } \\
\text { 3) }\end{array}$ & Normal (w5) & $\begin{array}{l}\text { Left frontoparietal infarction } \\
\text { (d4) }\end{array}$ & Normal (d3) & 21 & No & Normal & Normal & No \\
\hline 11 & Normal (d1) & Normal $(\mathrm{d} 3,7, \mathrm{w} 5)$ & Left parietal infarction (d4) & Normal (d7) & $12^{\star}$ & No & Normal & Normal & No \\
\hline 12 & Normal (d2) & Normal (d4) & $\begin{array}{l}\text { Left striatum/centrum } \\
\text { semiovale infarction }(\mathrm{d} 2)\end{array}$ & $\begin{array}{l}\text { Right asymmetrical } \\
\text { seizure activity (d2) }\end{array}$ & 24 & No & Normal & Normal & No \\
\hline
\end{tabular}

^ Further follow-information obtained from primary health care team; d, w: day or week of life on which investigation was performed.

Perinatal details of cases and controls were recorded from hospital notes, and compared using the $\chi^{2}$ for discrete, and Mann-Whitney tests for continuous, variables. Odds ratios (OR) were calculated when appropriate. Information concerning the neurodevelopmental status of the cases was obtained from the clinical notes, and was supplemented in some cases by updated information provided by general practitioners and health visitors.

Results

The total population of infants born at $>31$ weeks gestation in our hospital between 1987 and 1993 was 44518 . Over the same period, 100 babies of 32 weeks gestation or above $(0.22 \%)$ were investigated for neonatal seizures. A clear cause for the seizures was elicited in $85(85 \%)$; the relative prevalence of causes are shown in table 1. Hypoxic-ischaemic encephalopathy was the most common and neonatal cerebral infarction the second most common diagnosis. The 12 cases of neonatal cerebral infarction accounted for $12 \%$ of the total number of infants with seizures and 14\% of those with an established diagnosis.

All of the cases were at least of 37 weeks gestation. The characteristics of the 12 individual infants with neonatal cerebral infarction are shown in table 2. Eleven presented with seizures on the postnatal wards and were admitted to the nursery for investigation; one was admitted because of respiratory distress and subsequently had seizures. Convulsions were focal and clonic in eight infants, generalised and clonic in three, and the remaining infant had subtle seizures.

All babies were alert and responsive between episodes and were not encephalopathic. Onset of seizures was between 12 and 76 hours, with a median of 37 hours. This may reflect short postnatal confinements, because babies who had a first convulsion after discharge would not have been identified in this study. Computed tomography between days 2 and 8 of life was abnormal in all 12 infants, showing areas of low 
Table 4 Perinatal risk factors

\begin{tabular}{lll}
\hline & Neonatal cerebral infarction $(n=12)$ & Matched controls $(n=24)$ \\
\hline Maternal age & $31(22-40)$ & $28(18-38)$ \\
Abnormal pregnancy & $2 / 12$ & $4 / 24$ \\
Abnormal CTG & $5 / 9$ & $5 / 23$ \\
Meconium stained liquor & $6 / 12$ & $6 / 24$ \\
Spontaneous vaginal delivery & $6 / 12$ & $16 / 24$ \\
Umbilical artery pH & $7.19(7.02-7.34)$ & $7.21(7.01-7.38)$ \\
Apgar score at 1 minute & $6(2-9)$ & $8(4-10)^{\star}$ \\
Apgar score at 5 minutes & $9(8-10)$ & $10(8-10)$ \\
IPPV during resuscitation & $6 / 12$ & $3 / 24 \dagger$ \\
Male & $6 / 12$ & $12 / 24$ \\
Gestational age (weeks) & $40(37-42)$ & $40(36-42)$ \\
Birthweight $(\mathrm{g})$ & $3496(2541-4460)$ & $3673(2748-4707)$ \\
Admission age (hours) & $38(12-76)$ & $25(1-96)$ \\
Haemocrit on admission & $56(44-70)$ & $53(42-68)$ \\
Platelets $\left(\times 10^{9} / 1\right)$ & $234(103-345)$ & $274(129-413)$ \\
Glucose $(\mathrm{mmol} / \mathrm{l})$ & $3.6(1.6-4.6)$ & $3.5(1.6-5.0)$ \\
\hline
\end{tabular}

Mean and ranges displayed; * Mann-Whitney test $\mathrm{P}<0.05$; $\uparrow \chi^{2}$ test $\mathrm{P}<0.05$.

attenuation (table 3). Most lesions were peripheral, and in only one case was involvement of the basal ganglia reported. In 11 infants the computed tomogram abnormalities were unilateral. Case 8 was included, although there was a possible area of decreased attenuation in the right temporal lobe as well as the predominant left frontal lesion. Cerebral ultrasound scans showed unequivocal unilateral echodensities in only one infant when first scanned, and repeat scans showed asymmetrical echodensities in two others. Figs 1A-C show the abnormal computed tomogram and cerebral ultrasound scan from case 10 . Figure 2 shows the abnormal computed tomogram from case 1. Electroencephalographic (EEG) recordings were carried out on 11 cases, and were frankly abnormal in five (42\%), showing focal abnormalities in four. In most cases EEG and imaging results corresponded to each other and to the clinical features, but in two cases there was a discrepancy. Case 9 presented with left sided focal seizures: the computed tomogram showed an infarction in left middle cerebral artery territory, and the EEG was normal. Case 12 presented with left sided focal seizures and the EEG was consistent with asymmetrical and predominantly right sided abnormalities. However, the computed tomogram showed left cerebral infarction. In most cases seizures were easy to control, and in eight $(67 \%)$ there was a prompt response to an intravenous loading dose of $20 \mathrm{mg} / \mathrm{kg}$ phenobarbitone.

Perinatal risk factors for the 12 cases and the 24 controls matched for gestation, sex, and approximate date of birth, are compared in

Table 5 Prognosis of neonatal cerebral infarction in term infants (published data)

\begin{tabular}{|c|c|c|c|}
\hline Report & Year & $\begin{array}{l}\text { Proportion } \\
\text { impaired }\end{array}$ & Details \\
\hline Ment $^{3}$ & 1984 & $4 / 6$ & 1 hemiparesis, $1 \mathrm{sp}$. quad, 2 hypotonia \\
\hline Mantovani $^{2}$ & 1984 & $2 / 2$ & 2 hemiparesis \\
\hline Clancy $^{17}$ & 1985 & $2 / 11$ & 1 hemiparesis, 1 developmental delay \\
\hline Levy $^{6}$ & 1985 & $4 / 7$ & 4 hemiparesis \\
\hline Trauner $^{7}$ & 1986 & $2 / 8$ & 1 hemiparesis, 1 developmental delay \\
\hline $\operatorname{Sran}^{10}$ & 1988 & $5 / 14$ & 4 hemiparesis, 1 developmental delay \\
\hline Wulfeck $^{18}$ & 1991 & $9 / 14$ & 9 hemiparesis \\
\hline Fujimoto $^{9}$ & 1992 & $6 / 18$ & 5 hemiparesis, 1 developmental delay \\
\hline Perlman $^{8}$ & 1994 & $6 / 8$ & 5 hemiparesis, 1 hypotonia \\
\hline Bouza $^{19}$ & 1994 & $5 / 7$ & 5 hemiparesis \\
\hline Koelfen $^{13}$ & 1995 & $6 / 6$ & 6 hemiparesis, all with developmental delay \\
\hline Mercuri $^{4}$ & 1995 & $3 / 7$ & 3 hemiparesis \\
\hline
\end{tabular}

Only term infants who survived were considered for the outcome data quoted in this table. Infants with purely basal ganglia/thalamic lesions were omitted. table 4 . There were no significant differences between cases and controls with respect to maternal age, pregnancy complications, cardiotocographic abnormalities in labour, mode of delivery or umbilical cord acidosis. Cases had a lower Apgar score at 1 minute, and were more likely to require assisted ventilation for resuscitation (OR 7, 95\% CI $1.04-53.5$ ), but by 5 minutes Apgar scores ranged between 8 and 10 and were no different from those of controls. There were no differences between cases and controls in relation to birthweight, platelet count, haematocrit or plasma biochemistry on admission to the neonatal unit.

Table 3 shows the neurodevelopmental outcome of the 12 cases of neonatal cerebral infarction. Latest follow up data were recorded in the hospital notes at 11-60 (median 27) months of age - in 11 cases by consultants and in one case by a paediatric lecturer. Three children had been discharged from hospital follow up at 11,12 , and 14 months and attempts were made to determine their subsequent neurological outcome. The family of the child who was discharged with no neurological abnormalities at 11 months (case 9) had moved to America and could not be traced. Cases 8 and 11 (last seen in hospital at 14 and 12 months) had been regularly assessed by health visitors and their general practitioners and had no overt neurological impairment at 41 and 33 months of age. None of the infants had a persisting seizure disorder, none was causing any concern with respect to hearing or vision, and only one had a major motor deficit. Case 6 had a contralateral spastic hemiparesis but was developing normally in other respects. There was no evidence of cognitive deficits in any of the cases of neonatal cerebral infarction, although no formal testing was carried out. Parents were counselled in the postnatal period about the possibility of motor deficits in the future, but no specific interventions such as physiotherapy were initiated. The hemiparetic infant started treatment when physical signs became evident.

\section{Discussion}

Among our babies, neonatal cerebral infarction is the second commonest cause of seizures in neonates over 31 weeks gestation. Our finding that $12 \%$ of all neonatal seizures in full term 

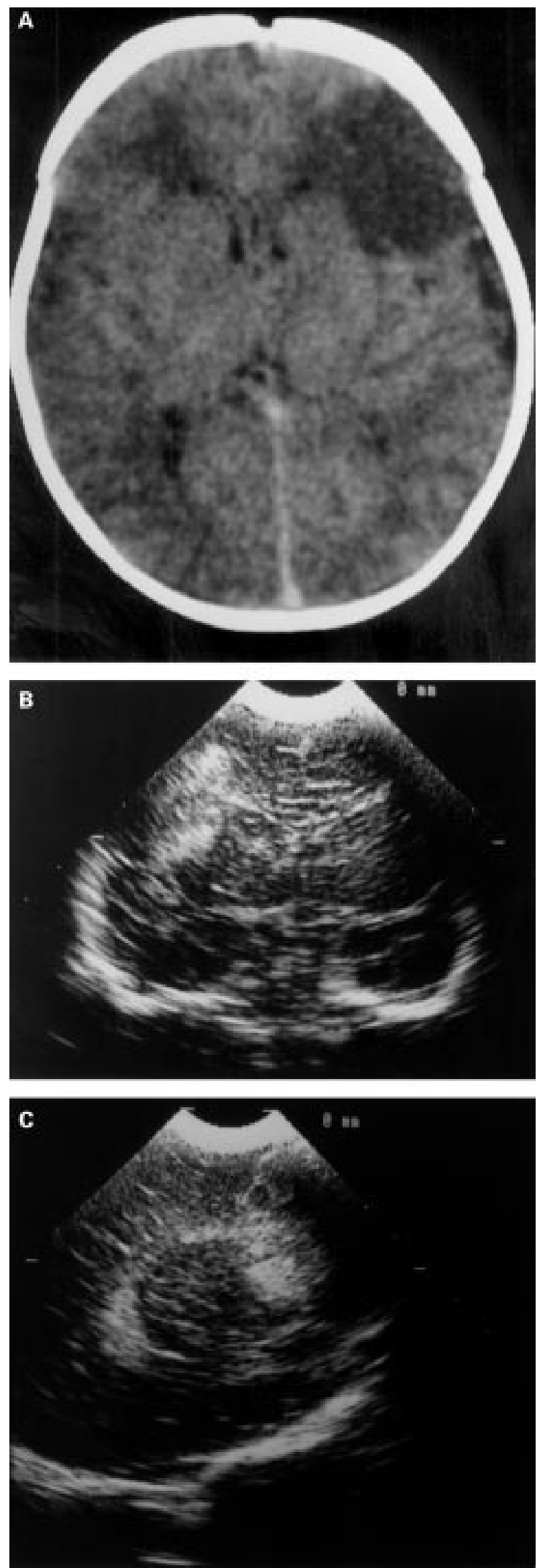

Figure 1 Computed tomogram on day $4(A)$; coronal and parasagittal ultrasound scans on day 3 (B) (C) from case 10. All show left frontal neonatal cerebral infarction. infants were due to neonatal cerebral infarction is similar to previously reported rates of $14 \%{ }^{6}$ and $17.5 \% .^{5}$ Over the period of data collection, there was an overall incidence of 11/44 518 $(0.025 \%)$ in our population. This is very similar to the reported incidence of $0.02 \%$ by Perlman. ${ }^{8}$ Our hospital population is not from a strictly defined geographical area and includes antenatal referrals but this would not have had a major influence on the prevalence calculation. We omitted the one case of neonatal cerebral infarction from outside our health district, and the effect of antenatal referrals on the denominator is small. This study and others have probably significantly underestimated the prevalence of neonatal cerebral infarction, because the diagnosis arises through imaging (usually computed tomography) which is only undertaken if there are seizures or other overt neurological signs. Many of the babies are well, without any evidence of neurological disturbance between seizures, so cases are likely to remain undetected if seizures do not occur, and may be missed or ignored if seizures are brief or subtle. Furthermore, the tendency towards short postnatal stays will lead to symptoms occurring at home and presentation to paediatricians and paediatric neurologists rather than neonatologists.

The varied and sometimes subtle symptomatology raise the possibility that some cases may not be detected in the perinatal period. If this is the case, neonatal cerebral infarction may be responsible for some cases of hemiplegic cerebral palsy in babies reported to have completely unremarkable perinatal histories. It is sometimes assumed that hemiplegic signs in infancy, which may be associated with contralateral computed tomography changes, are likely to be antenatal in origin unless there is a clearly documented history of perinatal insults. The computed tomograms in our cases showed acute changes rather than established atrophy, and the transient seizure disorders suggested an acute neurological event. Cerebral infarction can therefore occur in the few days before or after birth in healthy infants without overt underlying pathology.

This was not a prospective study, and the imaging and investigations were performed according to clinical need, rather than as part of a research protocol. Because the progress following initial discharge from hospital was usually benign, there was rarely an indication for a follow up computed tomogram. Sequential changes on magnetic resonance imaging (MRI) following neonatal cerebral infarction have recently been closely documented by Mercuri et al. ${ }^{4}$ MRI was not available to us during most of this period, and latterly we were reluctant to sedate or anaesthetise infants who were developing normally or who had a limited and predictable neurological deficit. We are therefore open to the criticism that the original computed tomography findings may have been misinterpreted, the diagnosis of cerebral infarction erroneous, and the good prognosis therefore much less surprising. However, all of our cases presented with clinical seizures, all the computed tomograms were reported by 

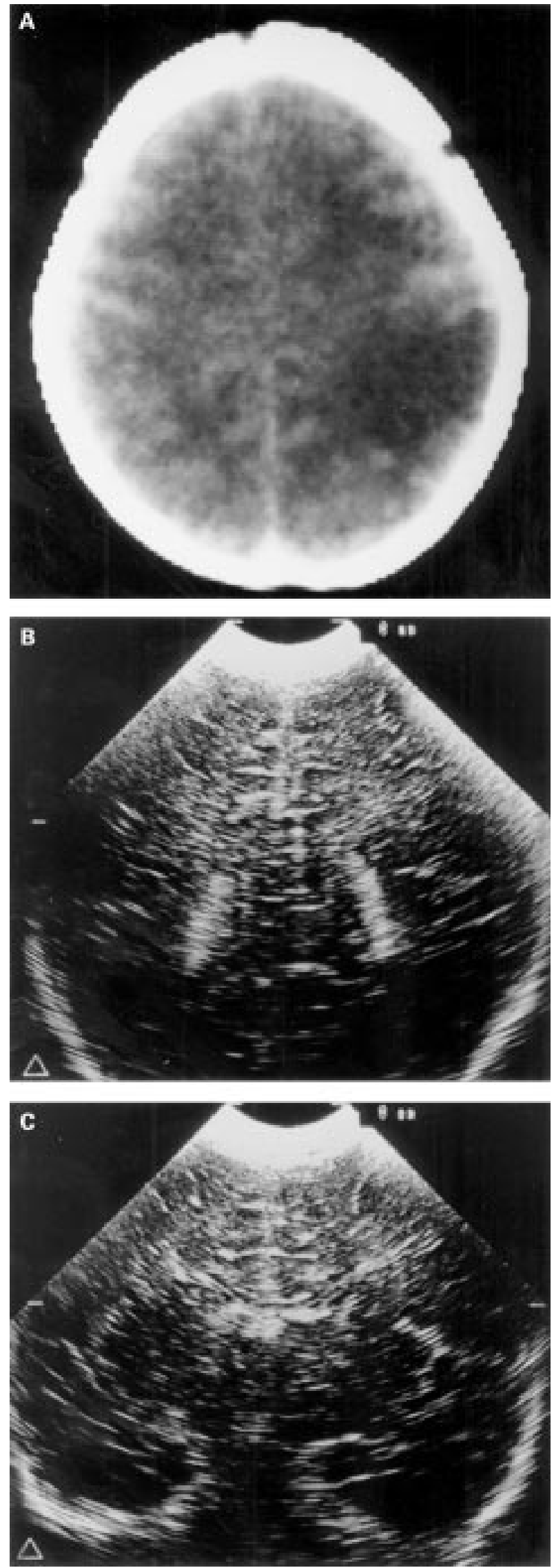

Figure 2 Computed tomogram on day 3(A) from case 1 showing left parietal neonatal cerebral infarction. Serial ultrasonography $(B),(C)$ revealed no changes in this case. consultant neuroradiologists, and table 3 shows that there was ancillary evidence of a focal cerebral lesion in many cases. A good outcome has been reported by others ${ }^{7} 910$ following neonatal cerebral infarct, and there has even been a recent suggestion that MRI shows cerebral "regeneration" following infarction to explain this. ${ }^{11}$ Follow up images are, of course, not available to clinicians advising parents in the neonatal period. Our finding of a good medium term prognosis following presentation with seizures and a computed tomographic diagnosis of neonatal cerebral infarction is therefore clinically relevant, even considering the constraints of a retrospective review and lack of follow up imaging.

Although periventricular and subcortical infarction may be visualised very well by cerebral ultrasonography, we found that scanning with 5 and $7.5 \mathrm{MHz}$ transducers was often unhelpful in the diagnosis of unilateral neonatal cerebral infarction. Ultrasound imaging may be normal even when interpreted in the light of information from a computed tomogram (fig 2). Others have suggested, like us, that ultrasonography has limitations in the diagnosis of neonatal cerebral infarction. ${ }^{5-12}$ However, Mercuri et $a l^{4}$ found that 11 of 14 cases of neonatal cerebral infarction detected by MRI were also evident on ultrasonography. Koelfen $e t a l^{13}$ found that all six infants with computed tomographic evidence of neonatal cerebral infarction had abnormal ultrasound scans, and Perlman et $a l^{6}$ used ultrasonography as a primary investigation and only proceeded to MRI if ultrasound scanning suggested neonatal cerebral infarction. Allen and Riviello ${ }^{14}$ reported that "we feel confident in US scanning to the point of not doing a CT scan in the face of negative serial US scans". Our data do not support this view.

The pathology of neonatal cerebral infarction is unknown, and few necropsy data are available because the babies rarely die. Some have suggested an embolic or thrombotic origin; these reports have been reviewed by Mannino and Trauner. ${ }^{1}$ Ment et $a l^{\beta}$ reported a high incidence of perinatal asphyxia and proposed an hypoxic-ischaemic origin, while others ${ }^{15}$ have implicated birth trauma. Some infarctions clearly correspond to the vascular distribution of a major cerebral artery-usually the middle cerebral - but this is not always the case. Because of the similarities in presentation, clinical course, and outcome, we included neonatal cerebral infarction of various sites and did not confine this report to so-called "middle cerebral artery" infarctions. We did not see the unexplained predominance of left sided lesions reported by other authors. ${ }^{6-8}$

Most of the published reports of neonatal cerebral infarction concern very small numbers of patients. Lien et $a \bar{l}$ have looked at risk factors for early onset neonatal seizures of any cause, but there are no previous published data comparing perinatal risk factors for neonatal cerebral infarction with controls. The case control data presented here concern a very small number of cases, and the analysis clearly has a very low power. As neonatal cerebral inf- 
arction is likely to have a multifactorial origin, it is not surprising that our study sheds very little light on possible aetiology. Resuscitation by assisted ventilation, either by "bag-and-mask" or endotracheal intubation, was more common in cases than in controls. In two cases endotracheal intubation was performed in the context of meconium stained liquor, and the umbilical cord artery $\mathrm{pH}$ was above 7.0 in all cases where it was measured. The postnatal course also suggests that the cases were not significantly asphyxiated as none had a low 5 minute Apgar score or a clinical course consistent with hypoxic-ischaemic encephalopathy. We used babies who had been admitted to the neonatal unit as controls because we wanted to compare haematological and biochemical results with cases. We felt it would be inappropriate to include as controls infants who had been admitted to the unit because of concerns about prolonged resuscitation, metabolic acidosis, or clinical suspicion of hypoxic-ischaemic encephalopathy. Our selection of "non-asphyxiated" controls will therefore have tended to increase any apparent excess of asphyxial markers in the cases of neonatal cerebral infarction.

Koelfen et $a l^{13}$ have reported a small series with a high incidence of assisted delivery, but this was not seen in our study. We have no evidence of cocaine abuse in any of the mothers of our cases, and none of the 12 was a twin. Polycythaemia has been implicated as a cause of neonatal cerebral infarction. ${ }^{16}$ Neonatal texts vary widely in their recommended threshold for dilutional partial exchange transfusion in asymptomatic polycythaemia, and one justification for an aggressive approach is the risk of "cerebral sludging." Interestingly, in our study neonatal cerebral infarction cases did not have a significantly higher mean haematocrit than controls. One baby with neonatal cerebral infarction had an initial haematocrit of 70 and received a dilutional exchange transfusion, but the rest all had an initial haematocrit of 60 or less.

The very low incidence of hemiplegic cerebral palsy at follow up was surprising. The parents were counselled that unilateral motor deficit of some degree was the most likely outcome. However, the previously published reports (summarised in table 5) show a wide range of outcomes.

de Vries and Levene ${ }^{12}$ summarised the data from published series and suggested that over $50 \%$ of infants with neonatal cerebral infarction are entirely normal at the age of 12-18 months, which is consistent with the data in table 5. However, our study shows significant motor deficit in one of 12 cases. One case was lost to follow up at 11 months but the rest were assessed at 20 months or more and judged to be normal, apart from one child with an obvious hemiparesis. This study confirms previous suggestions that cognitive development is unlikely to be seriously impaired following neonatal cerebral infarction. All of our cases, including the child with hemiparesis, were thought to have normal cognitive and sensory development when last seen, although they were not formally tested. Wulfeck et al reported that half of their cases showed a degree of language delay, although there was no significant delay in global cognitive function. ${ }^{18}$ Our study cannot exclude subtle cognitive or language deficits. We have established that 11 of 12 of these children are functioning without apparent disadvantage in the medium term, but problems may, of course, become apparent at school age. None has a persisting seizure disorder at present, although late recurrence of seizures after an eight year seizure free interval has been documented by Sran and Baumann. ${ }^{10}$

This seven year review of newborn infants admitted with seizures to a neonatal unit showed unilateral neonatal cerebral infarction to occur with a frequency of about 1 in 4000, and to be the second most common identifiable cause of seizures in infants of 32 weeks gestation and above. In our cases, the aetiology was usually unclear, and a small case-control study did not reveal any major risk factors for neonatal cerebral infarction. The prognosis in our patients was more benign than has previously been reported.

We thank Drs Andrew Wilkinson, Kevin Ives, and Maura Mcdonnell for permission to report their patients.

Dr Javier Estan was supported by a grant FIS 95/5493 from the Spanish Health Service.

1 Mannino FL, Trauner DA. Stroke in Neonates. $\mathcal{f}$ Pediatr 1983;102;605-10.

2 Mantovani JF, Gerber GJ. "Idiopathic" neonatal cerebral infarction. Am f Dis Child 1984;138:359-62.

3 Ment LR, Duncan CC, Ehrenkranz RA. Perinatal cerebral infarction. Ann Neurol 1984;16:559-68.

4 Mercuri E, Cowan F, Rutherford M, Acolet D, Pennock J, Dubovitz L. Ischaemic and haemorrhagic brain lesions in newborns with seizures and normal Apgar scores. Arch Dis newborns with seizures
Child 1995;73:F67-74.

5 Lien JM, Towers CV, Quilligan EJ, de Veciana M, Toohey JS, Morgan MA. Term early-onset neonatal seizures: Obstetric characteristics, etiologic classifications and perinatal care. Obstet Gynecol 1995;85:163-9.

6 Levy SR, Abroms IF, Marshall PC, Rosquete EE. Seizures and cerebral infarction in the full term newborn. Ann Neurol 1985;17:366-70.

7 Trauner DA, Mannino FL. Neurodevelopmental outcome after neonatal cerebrovascular accident. $\mathcal{f}$ Pediatr 1986;108:459-61.

8 Perlman JM, Rollins NK, Evans D. Neonatal stroke: Clinical characteristics and cerebral blood flow velocity measurements. Pediatr Neurol 1994;11:281-4

9 Fujimoto S, Yokochi K, Togari H, Nishimura Y, Inukai K, Futamara M, et al. Neonatal cerebral infarction: Symptoms, CT findings and prognosis. Brain Dev 1992;14:4852.

10 Sran SK, Baumann RJ. Outcome of neonatal strokes. Am $\mathcal{F}$ Dis Child 1988;142:1086-8.

11 Rutherford MA, Pennock JM, Cowan FM, Dubovitz LMS, Hajnal J, Saeed N, et al. Serial magnetic resonance imaging of the brain following neonatal infarction: signs of regeneration. Early Hum Dev 1996;46:266-7.

12 de Vries LS, Levene MI. Cerebral ischaemic lesions. In:Levene MI, Lilford RJ, eds. Fetal and neonatal neurology and neurosurgery. 2nd edn. Edinburgh: Churchill Livingstone, 1995:379-82

13 Koelfen W, Freund M, Varnholt V. Neonatal stroke involving the middle cerebral artery in term infants: Clinical presentation, EEG and imaging study, and outcome. Dev Med Child Neurol 1995;37:204-12.

14 Allan WC, Riviello JJ. Perinatal cerebrovascular disease in the neonate: Parenchymal ischemic lesions in term and preterm infants. Ped Clin North Am 1992;39:621-50.

15 Roessmann U, Tyler Miller R. Thrombosis of the middle cerebral artery associated with birth trauma. Neurology 1980;30:889-92.

16 Amit M, Camfield PR. Neonatal polycythaemia causing multiple cerebral infarcts. Arch Neurol 1980;37:109-10.

17 Clancy R, Malin S, Laraque D, Baumgart S, Younkin D. Focal motor seizures heralding stroke in full-term

18 Wulfeck BB, Trauner DA, Tallal PA. Neurological, cognitive and linguistic features of infants after early stroke. Pediatr Neurol 1991;7:266-9.

19 Bouza H, Dubovitz LMS, Rutherford M, Cowan F, Pennock JM. Late magnetic resonance imaging and clinical findings in neonates with unilateral lesions on cranial ultrasound. Dev Med Child Neurol 1994;36:951-64. 